\title{
Common consults
}

\section{How am I going to perform the caesarean?}

\section{Angelika Von Heimendahl}

Caesarean sections are either emergency procedures, a decision made after prolonged gestation or an elective procedure. The lecture will give some ideas of set up and procedures for the different scenarios

\section{EMERGENCY PROCEDURE}

Much of the advice given to owners preoperatively cannot be applied in this case. It is important to make sure that the dates (ovulation testing and matings) given by the breeder are accurate and it is always worth checking on your own calendar before performing this operation. Any request from breeders below 61 days' gestation should be resisted. The most common reasons for emergency Caesarean sections are secondary inertia and dystocia, both of which will be discussed.

If possible, try to get as many hands on deck as possible for the part of the Caesarean when the puppies are produced, as active resuscitation is an important part of good survival rates.

\section{PROLONGED GESTATION}

This term is often used to for bitches that have not had blood progesterone tested so the true length of gestation is not known. In this case it is difficult to distinguish between primary inertia or longer gestation due to sperm survival after mating and later fertilization. The lecture will give some help with decision making, blood testing ultrasonography and radiography.

\section{ELECTIVE CAESAREANS}

Given the price for some puppies of brachiocephalic breeds there is more pressure on veterinary surgeons to perform Caesarean sections than ever before. The lecture will touch on some of the ethical questions, Kennel Club regulations and advice for breeders. Elective Caesareans may also be performed for a range of other reasons, when they can be a lifesaving intervention.

\section{KEY LEARNING OBJECTIVES}

- Know when to choose to perform a Caesarean section

- Understand what is needed to have the best outcome

- Understand the implications for future breeding

\section{MULTIPLE CHOICE QUESTIONS}

1. A Golden Retriever bitch that has been scanned in pup with 12 puppies has given birth to three puppies over the last 8 hours The last puppy was born 4 hours ago. How should you proceed with management of this case?

(A) Give multiple low-dose injections of oxytocin (2-3 IU) and deliver the puppies one by one

(B) As the cervix is clearly open the chances of delivering the other puppies are good

(C) An immediate Caesarean section is the best chance for delivering the other nine puppies alive

(D) Wait to perform a Caesarean section until the oxytocin injections stop working

2. A Newfoundland bitch, confirmed pregnant by another practice/scanner comes to the surgery 63 days after mating. Mating occurred on the right day after ovulation testing. The owner reports that only one foetus was visible on ultrasonography. He wants a Caesarean as he has read on the internet that singletons often don't induce birth sufficiently. What should you do?

(A) You check the results of the progesterone test and the mating date and after you are satisfied that these are correct perform a Caesarean section.

(B) You radiograph the bitch to make sure she is pregnant

(C) You test a blood sample for progesterone to make sure it is below $2 \mathrm{ng} / \mathrm{l}$ indicating she has come to the end of gestation

(D) You check the mammary glands, which are slightly enlarged and leave the bitch another 24 hours to start labour

3. A bitch, confirmed pregnant with eight puppies, is showing greenish-black vaginal discharge on day 52 of her pregnancy. What would you do?

(A) Ultrasonography to confirm heartbeats and if there are live foetuses wait to enable them to go full term

(B) Uteroverdin is a sign that the pregnancy has been terminated and aglepristone should be given to empty the uterus to avoid foetal remnants and infection

(C) Once the first puppy has been born and parturition does not progress a Caesarean section should be performed

(D) If the ultrasound scan confirms that all the foetuses have died a Caesarean should be performed immediately 